\title{
Morphological and genetic perspectives of hybridization in two contact zones of closely related species of Petunia (Solanaceae) in southern
} Brazil

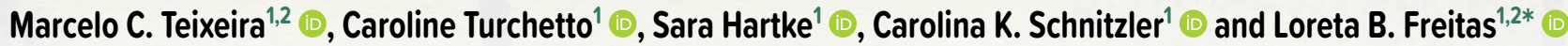

Received: April 16, 2019

Accepted: July 8, 2019

\begin{abstract}
Interspecific hybridization has several consequences for parental species, from blurring species limits to the emergence of new taxa. Petunia axillaris and P. exserta occur in sympatry in southernmost Brazil and naturally hybridize despite their different pollination syndromes and habitat requirements. We employed genetic and morphological analyses to characterize two contact zones between the species with the aim of determining the effect of interspecific hybridization. Microsatellite loci and a morphometric evaluation of the corolla shape were used to classify individuals based on their origin as pure parental or hybrids. Corolla color was used to classify individuals a priori (white, red or intermediate, for P. axillaris, P. exserta or hybrid, respectively). Corolla color was found to be a good indicator of the genetic component of each species and their hybrid, while the shape of the corolla did not always correspond to genetic origin. Hybridization increased the variability, and introgression occurred in both directions in this system.
\end{abstract}

Keywords: genetic diversity, hybridization, introgression, morphometric analysis, Petunia

\section{Introduction}

Contact zones between closely related species are used to investigate species boundaries, barriers against interspecific gene exchange, and consequences of hybridization (Cinget et al. 2015). Genetic ancestry can provide information on the origin of the individuals or populations, while morphological data may provide information about the hybridization phenomena (Szlachetko et al. 2017).

In Serra do Sudeste, which is in the middle of the Pampas region in south Brazil, we can find two herbaceous and annual species of the Petunia genus (Solanaceae). These species are closely related (Reck-Kortmann et al. 2014) and share several morphological traits (Stehmann et al.
2009) and genetic polymorphisms (Lorenz-Lemke et al. 2006). Despite their evolutionary proximity and growth in a sympatric area, these species preserve their genetic boundaries (Segatto et al. 2014), and prezygotic barriers probably contribute to the prevention of interspecific crosses (Turchetto et al. 2015a; b).

Petunia axillaris subsp. axillaris is widely distributed across the Pampas (Turchetto et al. 2014), whereas P. exserta Stehmann is endemic to the Guaritas formation (Segatto et al. 2014) in Serra do Sudeste. The Guaritas region is a rock formation that is characterized by sandstone towers of 200 - 500 meters in elevation and harbors complex open field vegetation (Overbeck et al. 2007). Petunia axillaris subsp. axillaris (hereafter $P$. axillaris) inhabits the sunny top and faces of these towers, while P. exserta is found

1 Laboratório de Evolução Molecular, Departamento de Genética, Universidade Federal do Rio Grande do Sul, 91501-970, Porto Alegre, RS, Brazil 2 Programa de Pós-Graduação em Botânica, Universidade Federal do Rio Grande do Sul, 90509-900, Porto Alegre, RS, Brazil

* Corresponding author: loreta.freitas@ufrgs.br 
inside the small cavities in the rocks where individuals grow fully protected from sunlight and rain. These two Petunia species diverge on their pollination syndromes and display many floral traits related to attracting their putative main pollinators. The white, scented, UV-absorbent flowers of $P$. axillaris preferentially attract the hawkmoths Manduca sexta (Venail et al. 2010; Klahre et al. 2011), whereas the bright red corollas with the exserted stamens of $P$. exserta flowers constitute common traits that are present in several birdpollinated species (Proctor et al. 1996; Gübitz et al. 2009). These morphological differences and ecological peculiarities could indicate the isolation between these sympatric species; however, molecular evidence suggests that interspecific hybridization occurs during each reproductive season (Lorenz-Lemke et al. 2006; Segatto et al. 2014; Turchetto et al. 2015b; 2019).

Numerous populations of these two species can be found throughout the Serra do Sudeste; in many towers, it is possible to observe patches of individuals of each species growing nearby (Segatto et al. 2014; Turchetto et al. 2014). Especially in two of these sites (CO1 and CO2), there are short spatial differences between plants with $P$. axillaris morphology on the top and faces of the towers and the typical P. exserta and intermediary colored individuals that grow inside the cavities. Based only on corolla color, these individuals with flowers ranging from pale to dark pink were considered as putative hybrids; considering the spatial distribution and the plastid haplotype sharing among individuals from the same tower, they were proposed as vectors of introgression for P. exserta (Lorenz-Lemke et al. 2006). Posteriorly, these same individuals were analyzed for nuclear markers, which confirmed their hybrid condition (Turchetto et al. 2015b). A more comprehensive sample in Serra do Sudeste for P. exserta revealed that individuals with intermediary corolla colors can also be found in other towers, but in contrast to those collected from $\mathrm{CO} 1$ and $\mathrm{CO} 2$, these individuals constitute a second evolutionary lineage of $P$. exserta (Turchetto et al. 2019). Corolla shape is different between species based on geometric morphometrics ( $M C$ Teixeira unpubl. res.).

In this study, we explored the species boundary in the $\mathrm{CO} 1$ and $\mathrm{CO} 2$ sites through an integrative approach combining genetic and morphological evaluations for all adult individuals that can be found during one flowering season. Our main goal was to characterize the two contact zones between $P$. axillaris and P. exserta to identify the effect of interspecific hybridization on the species. Specifically, we aimed to verify whether intermediary colored individuals correspond to hybrids plants and to estimate the contribution of genetic profiles and morphological shape to correctly classify these individuals. To these aims, we utilized microsatellite markers to characterize genetic differentiation and modeled the floral shape for the same individuals classified a priori as $P$. axillaris, P. exserta or putative hybrids based on their corolla color.

\section{Materials and methods}

\section{Sampling and DNA extraction}

We sampled all of the adult individuals that were found in the two sites, $\mathrm{CO} 1\left(30^{\circ} 53^{\prime} 48.153^{\prime \prime} \mathrm{S} 53^{\circ} 25^{\prime} 16.080^{\prime \prime} \mathrm{W}\right)$

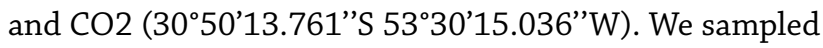
individuals during the 2015 flowering season, and all individuals were collected at the same time and in the same phenophase (open flower immediately after the anthesis). Vouchers were taken to record each collection site and phenotype and were deposited at ICN herbaria (P. axillaris: ICN185145; P. exserta: ICN185146; Hybrids: MCT003, МCT004, МСT005, МСТ006, and МСТ007). The plants were classified based on a visual assessment of corolla color (Tab. S1 in supplementary material) as P. axillaris (pure white flowers), P. exserta (dark and bright red corollas), and putative hybrids (intermediary colored flowers ranging from pale to dark pink).

Four or five young and fresh leaves per individual were collected, stored in silica gel, and powdered in liquid nitrogen. The total genomic DNA was extracted using a CTAB (cetyl-trimethyl ammonium bromide)-based protocol (Roy et al. 1992). DNA quality and concentration were evaluated through electrophoresis on an agarose gel stained with GelRed ${ }^{\circledR}$ (Biotium Inc., Freemont, USA) and in a Nanodrop spectrophotometer (Thermo Fischer Sci. Co., Waltham, USA).

\section{Genetic data acquisition}

We genotyped all the individuals using seven nuclear microsatellite (SSR) previously published (Bossolini et al. 2011) and broadly used to evaluate genetic diversity in Petunia species (Turchetto et al. 2015b) loci (PM8, PM21, PM167, PM173, PM177, PM188, and PM195). The PCR reactions were performed according to Turchetto et al. (2015b). The DNA fragments were denatured and sizefractionated utilizing capillary electrophoresis on a 3500 DNA Analyzer (Applied Biosystems CO., Forester City, USA) using the GeneScan 500 LIZ (Applied Biosystems) pattern. Genemarker Demo 1.97 (Softgenetics, State College, USA) software was applied to determine the number of alleles per locus with manual inspections and reference samples for P. axillaris and P. exserta (Turchetto et al. 2015b) in each run. Genotyping errors from stutter bands, allele dropout, and null alleles were verified with Micro-Checker software (Oosterhout et al. 2004).

\section{Summary statistics}

We estimated the number of alleles $(\mathrm{N})$, number of private alleles (E), allele richness (R), genetic diversity (GD), and inbreeding coefficient (FIS; Weir \& Cockerham 1984) per loci via Fstat 2.9.3.2 (Goudet 1995). We also estimated 
the observed (Ho) and expected (He) heterozygosities under Hardy-Weinberg equilibrium (HWE) after Bonferroni correction and analysis of molecular variance (AMOVA; Excoffier et al. 1992) with 10.000 permutations using Arlequin 3.5.1.2 (Excoffier \& Lischer 2010). We compared the diversity indices per collection site through ANOVA using the R 0.7.6 dplyr package (available at https:// CRAN.R-project.org/package $=$ dplyr .

\section{Genetic clustering and admixture}

We estimated the levels of hybridization and the proportion and direction of introgression using model-based Bayesian clustering employing Structure 2.3.2 software (Pritchard et al. 2000) assuming an admixture model and correlated allelic frequencies. Each run was conducted with 250.000 burn-in periods and 1.000.000 Markov chain Monte Carlo (MCMC) repetitions after burn-in. Ten independent runs were performed, and the results were examined for convergence across runs. The number of groups was estimated as the most likely number of genetic clusters using the maximum value of $\Delta K$ (Evanno et al. 2005), as implemented in Structure Harvester 0.6.93 (Earl \& Holdt 2012), and the estimate of $\operatorname{Pr}(X \mid K)$ was calculated from Structure. Structure was also used to classify individuals as parental species or hybrids, as proposed in Burgarella et al. (2009). Individuals with $q \geq 0.90$ were classified as $P$. axillaris; those with $q \leq 0.10$ were classified as $P$. exserta; and all that showed intermediary values $(0.10<q<0.90)$ were considered hybrids.

We also performed a Bayesian clustering analysis that was implemented in Newhybrids 1.1 software (Anderson \& Thompson 2002) to assign individuals into genotypic classes according to their genetic composition and based on the total probability $(Q)$ of each one to belong to a specific class: P. axillaris (parental 1), P. exserta (parental 2), firstgeneration interspecific hybrid (F1), second generation hybrid (F2), backcross with P. axillaris (BC1), and backcross with P. exserta (BC2). We ran two independent analyses using Jeffrey's priors with uniform priors that included 100.000 steps as burn-in followed by $1.000 .000 \mathrm{MCMC}$ interactions that were performed to assure the convergence of chains and homogeneity across runs. Analyses were performed without previous information on populations or taxonomic identity (Vähä \& Primmer 2006).

\section{Concordance between genotype and flower shape}

We compared the genetic profiles between individuals considering the Bayesian analyses with their respective floral shape based on the geometric morphometric analysis. We used a digital photograph of one flower per plant in the planar position of the corolla with 15 of the landmarks that were obtained from MC Teixeira unpubl. res., and we modeled the corolla shape using MorphoJ package (Klingenberg 2011) and analyzed the data with principal components (PCA) and discriminant function (DFA) analyses. We also visually inspected the concordance between corolla color and floral shape.

\section{Results}

\section{Color classes}

We found 22 adult individuals in $\mathrm{CO} 1$ and 24 in $\mathrm{CO} 2$ during the flowering season in 2015. Individuals were classified according to corolla color, and differences were observed in each site. From CO1, 16 individuals showed white flowers and were classified as P. axillaris (A); only one individual displayed a red and bright corolla and was identified as $P$. exserta (E); five individuals were intermediary colored and were designated as putative hybrids $(\mathrm{H})$. Among the 24 individuals that were collected from $\mathrm{CO} 2$, we classified eight individuals as P. axillaris, seven as P. exserta, and nine as putative hybrids (Tab. S1 in supplementary material).

\section{Genetic diversity}

All of the SSR loci were polymorphic among individuals and collection sites (Tab. 1). Private alleles were found in both sites, and allele numbers per locus were usually similar between sites. The total GD per site was higher in CO1 than in $\mathrm{CO} 2$, and neither site was in HWE because the excess of homozygotes, which produced high and significant FIS values. Inbreeding as estimated through FIS was higher in $\mathrm{CO} 2$ than in $\mathrm{CO} 1$. The AMOVA results showed that most of the genetic variability exists within the populations (FST $=0.21 ; \mathrm{P}<0.05$ ).

\section{Genetic clustering and admixture}

Generally, the Bayesian analyses assignment were concordant with individuals' classification based on corolla color (each species in Structure and purebred in Newhybrids). The same concordance was not observed considering the putative hybrids (hybrids in Structure and F1 or F2 in Newhybrids for intermediary colored individuals).

The best number of groups according to the $\Delta K$ (Evanno et al. 2005) in the Structure analysis was $K=2$, which mostly corresponded to each species (Fig. 1). The two genetic components were present in both sites. From CO1, just one individual (A748) that was visually classified as $P$. axillaris did not show a score of $q \geq 0.90$, and only one P. exserta individual (E711) showed a $q \leq 0.10$. Based on this $q$ threshold, just one individual from $\mathrm{CO} 1$ (H745) was classified as a putative hybrid and showed an intermediate genetic component $(0.10<q<0.90)$, whereas the remaining four had the P. exserta genetic component. A748 had white corollas and a $q=0.5$ and, according to Structure, would be a hybrid. All of the individuals from $\mathrm{CO} 2$ that were classified 
as $P$. axillaris based on their corolla color showed a $q \geq 0.90$, and those displaying red flowers (P. exserta) had $q \leq 0.10$. Two of the putative hybrids showed intermediate scores $(0.10<q<0.90)$, while one had a P. axillaris component (H740), and four others showed a P. exserta component.

In the Newhybrids analysis (Fig. 2) we did not find evidence for $\mathrm{F} 1$ hybrids in either site, and most of the plants $(22 / 24)$ that were identified as $P$. axillaris or P. exserta based on their corolla color were purebred individuals of each respective species. Two of the plants that were classified as $P$. axillaris (A748 and A794) and two others that were identified as $P$. exserta (E711 and E774) based on corolla color were classified as F2 hybrids. The putative hybrids based on their floral color from $\mathrm{CO} 1$ were classified as purebred $P$. exserta (four individuals) or as segregating F2 (one plant), whereas putative hybrid plants from $\mathrm{CO} 2$ based on corolla color were identified as segregating F2 (three plants), purebred $P$. axillaris (one individual) or purebred $P$. exserta (five plants). The Structure and Newhybrids analyses were discordant and assigned only four individuals (H745 from CO1 and A794, E774, and H742 from CO2).

\section{Concordance between genotype and flower shape}

The first two PCA (Fig. 3; Tab. S2 in supplementary material) explained more than $50 \%$ of the variation in

Table 1. Genetic diversity indices per collection site, $\mathrm{CO} 1$ and $\mathrm{CO} 2$, respectively, considering seven microsatellite loci. Pop - population code; $\mathrm{Cr}$ - chromosomal location; SR - variation in size of alleles in base pairs; $\mathrm{E}$ - number of private alleles; $\mathrm{N}$ - total number of alleles; GD - genetic diversity; R - allelic richness; Ho - heterozygosity observed; He - expected heterozygosity under Hardy-Weinberg equilibrium; FIS - coefficient of inbreeding; * significant values of Hardy-Weinberg equilibrium deviation after Bonferroni correction (P <0.05).

\begin{tabular}{|c|c|c|c|c|c|c|c|c|c|c|}
\hline Pop & Locus & $\mathrm{Cr}$ & SR & $\mathbf{E}$ & $\mathbf{N}$ & GD & $\mathbf{R}$ & Ho & $\mathrm{He}$ & $F_{\text {IS }}$ \\
\hline \multirow{7}{*}{ CO1 } & PM188 & I & $124-142$ & 1 & 6 & 0.73 & 6 & 0.57 & $0.73^{*}$ & 0.22 \\
\hline & PM195 & I & $193-214$ & 1 & 5 & 0.71 & 5 & 0.09 & $0.69^{*}$ & 0.87 \\
\hline & PM21 & II & $125-131$ & 1 & 3 & 0.55 & 3 & 0.38 & $0.55^{*}$ & 0.31 \\
\hline & PM8 & IV & $163-183$ & 2 & 4 & 0.72 & 4 & 0.27 & $0.71^{*}$ & 0.62 \\
\hline & PM173 & IV & $166-196$ & 2 & 5 & 0.78 & 5 & 0.45 & $0.77^{*}$ & 0.42 \\
\hline & PM167 & $\mathrm{V}$ & 279-309 & 6 & 9 & 0.77 & 9 & 0.31 & $0.76^{*}$ & 0.59 \\
\hline & PM177 & $\mathrm{V}$ & $212-258$ & 4 & 11 & 0.9 & 11 & 0.52 & $0.89^{*}$ & 0.42 \\
\hline \multicolumn{4}{|c|}{ Total } & 17 & 43 & 0.74 & 6 & 0.37 & $0.73^{*}$ & $0.49^{*}$ \\
\hline \multirow{7}{*}{$\mathrm{CO} 2$} & PM188 & I & $124-142$ & 2 & 5 & 0.56 & 5 & 0.31 & $0.55^{*}$ & 0.43 \\
\hline & PM195 & I & $190-214$ & 1 & 5 & 0.67 & 5 & 0.20 & $0.66^{*}$ & 0.69 \\
\hline & PM21 & II & $119-128$ & 1 & 3 & 0.51 & 3 & 0.20 & $0.50^{*}$ & 0.59 \\
\hline & PM8 & IV & $175-179$ & 0 & 2 & 0.50 & 2 & 0.16 & $0.49^{*}$ & 0.66 \\
\hline & PM173 & IV & 166-199 & 2 & 5 & 0.61 & 5 & 0.29 & $0.60^{*}$ & 0.52 \\
\hline & PM167 & $\mathrm{V}$ & 291-306 & 1 & 4 & 0.58 & 4 & 0.28 & $0.57^{*}$ & 0.51 \\
\hline & PM177 & $\mathrm{V}$ & $212-256$ & 5 & 10 & 0.82 & 10 & 0.39 & $0.81^{*}$ & 0.52 \\
\hline \multicolumn{4}{|c|}{ Total } & 12 & 34 & 0.61 & 5 & 0.26 & $0.60^{*}$ & $0.56^{*}$ \\
\hline
\end{tabular}

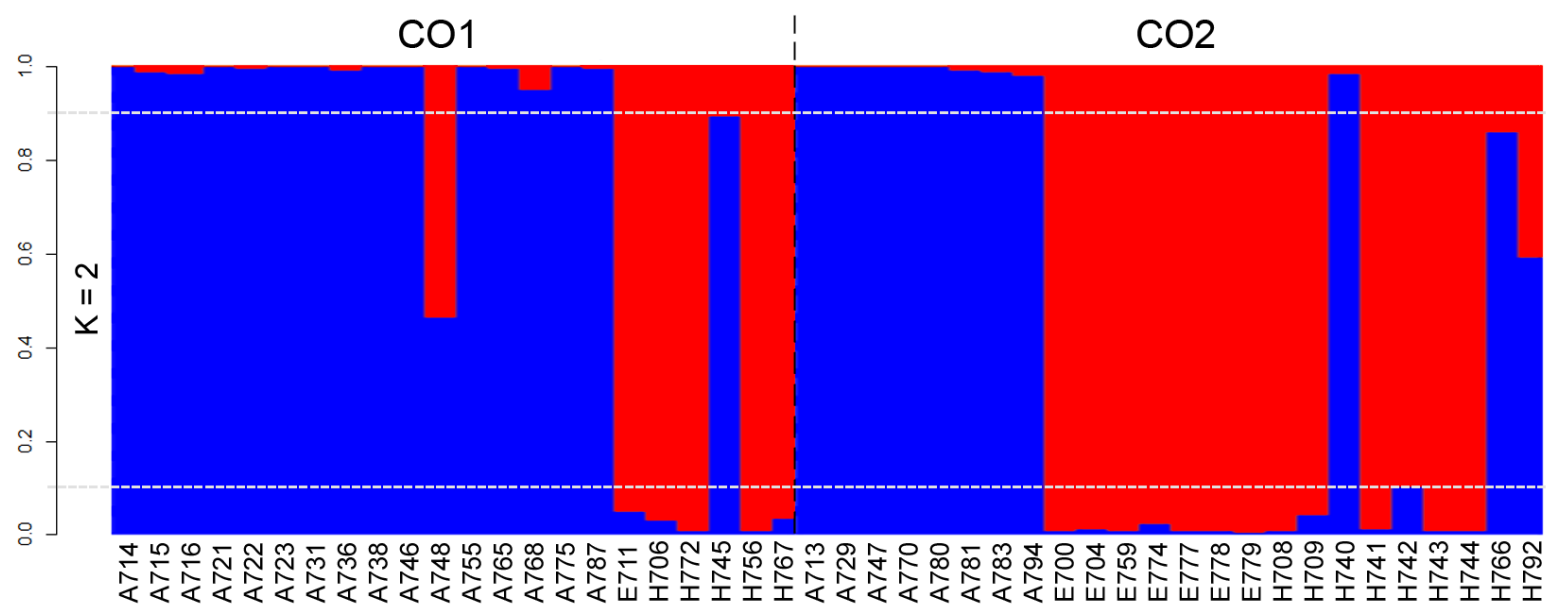

Figure 1. STRUCTURE bar plot under admixture coefficients model based on seven microsatellite loci and 46 adult individuals of Petunia exserta, P. axillaris, and their putative hybrids classified based on corolla color. Each bar represents individuals, and blackdotted vertical line indicates each collection site, $\mathrm{CO} 1$ and $\mathrm{CO} 2$, respectively; different colors correspond to genetic components $(\mathrm{K}=2)$ and individuals' membership. Horizontal white-dotted lines delimit q threshold. Individuals' code follows color groups (A - white; E - red; H - pink shades). 


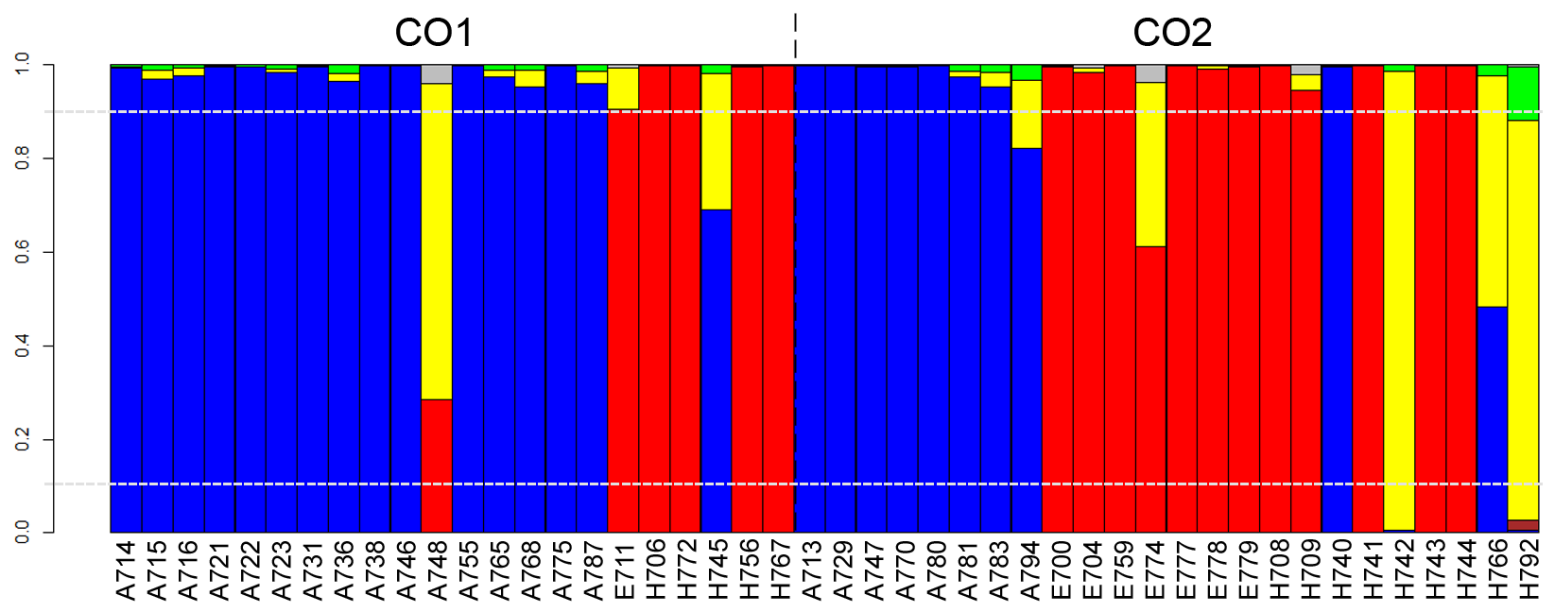

Figure 2. Posterior probabilities (Q) for all analysed plants using NewHybrids assigned to six classes following the legend for pure parental species (P. axillaris - blue; or P. exserta - red), F1 (brown), F2 (yellow), backcrosses with P. exserta (grey), and backcrosses with P. axillaris (green). Black-dotted vertical line indicates each collection site, $\mathrm{CO} 1$ and $\mathrm{CO} 2$, respectively, and horizontal white-dotted lines delimit $Q$ threshold. Individuals' code follows color groups (A - white; E - red; $\mathrm{H}$ - pink shades).

corolla shape for these individuals that correspond to a pentagonal corolla (predominantly observed in P. axillaris) or a star like shape (P. exserta) in the planar view. Considering the $95 \%$ confidence intervals (Fig. S1 in supplementary material), we observed three main groups that were preferentially composed of white, red, and intermediary colored corollas. Some superimpositions were observed mainly among the putative hybrids and $P$. exserta individuals, and none were observed for the P. axillaris and P. exserta color-based classification. Five of the individuals that were classified as P. axillaris based on their corolla color occupied the morphospace of putative hybrids, whereas only two intermediary colored individuals were observed in the $P$. axillaris morphospace. Based on the DFA, intermediary colored individuals had a different corolla shape when compared to red colored flowers $(0.08 ; \mathrm{P}<0.05)$ or white corollas $(0.07 ; \mathrm{P}<0.05)$. White and red flowers displayed different morphologies $(0.13 ; \mathrm{P}<0.05)$.

The results of the Structure and Newhybrids analyses did not agree with flower shape in the classification of 13 individuals, five of which were from $\mathrm{CO} 1$ and eight from $\mathrm{CO} 2$. In these cases, floral morphology and color agreed four times (A748 from CO1; A794, E774, and H740 from $\mathrm{CO} 2$ ) and disagreed in relation to the classification of the individual H706 (CO1), which is a putative hybrid based on its color but displays the corolla shape of $P$. exserta. Eight times, plants showed an intermediary corolla color and also displayed an intermediary corolla shape that was within the $95 \%$ confidence interval for the P. exserta morphospace.

\section{Discussion}

Hybridization plays an essential role in plant evolution (Abbott et al. 2016), and secondary contact zones between recently diverged species provide a unique opportunity to study the effect of hybridization on genetic and morphological diversity (Meier et al. 2016).

The consequences of interspecific hybridization may vary profoundly among the involved species and populations, from the elimination of species' limits, thus transforming two species in one (Soltis \& Soltis 2009), to the generation of new adaptations, which promotes speciation (Meier et

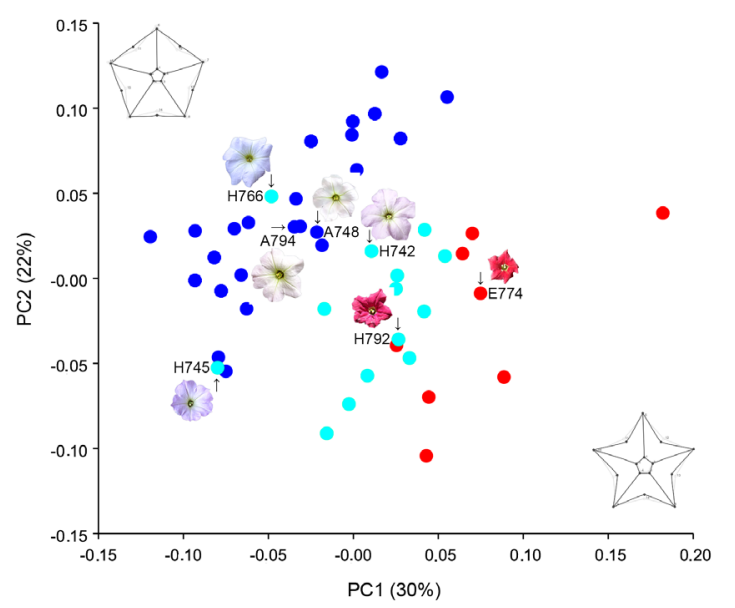

Figure 3. Principal Components Analysis (PCA) of the Petunia axillaris, P. exserta, and their putative hybrids, obtained by 15 landmarks from one photograph per specimen in a planar view. Individual are represented by a dot, colored according to the group to which it belongs ( $P$. axillaris - blue; $P$. exserta - red; putative hybrids - cyan). The axes are the principal components 1 and 2 , respectively, which represent changes in floral shape. Black arrows indicate dots corresponding to individuals assigned as hybrids based on their genetic components. The pentagonal form represents $P$. axillaris floral shape and star-like represents $P$. exserta corolla morphology. 
al. 2016). Our genetic data confirmed the hybrid nature of prior-classified individuals based on corolla color and demonstrated that, in two secondary contact zones between two closely related species that inhabit different microenvironments, interspecific hybridization and introgression regularly occur.

Hybridization is the basis of diversity in angiosperms (Yakimowski \& Rieseberg 2014) and is responsible for the emergence of many closely related species that diverge by their floral syndromes and may or may not maintain their ability to interbreed (Imbert et al. 2014; Cronk \& Yang 2016; Milano et al. 2016). The two studied Petunia species have maintained their morphological and genetic limits, but the number of hybrids in both microenvironments is growing, which changes the corolla shape and color and the prevalence of genetic polymorphisms in both parental species. Alternatively, we could have identified a stable hybrid lineage that reproduces in the contact zones in both parental habitats, which independently segregates neutral genetic markers and floral morphology.

Herein, we described the genetic and morphological diversity between two Petunia species that diverge mainly in their pollination syndromes and ecological adaptations (Stehmann et al. 2009). Hybridization between them promoted the appearance of a new class of color and corolla shape, as seen in PCA analyses of flower morphology, and this was especially prevalent inside rock cavities. Although they were sometimes dissociated (see Structure results), changes in color and shape followed genetic polymorphism shifts and brought new morphological trait combinations to the $P$. exserta environment. These atypical individuals can compete with the canonical ones for pollinators, among other resources.

In Petunia species, a few mutations can lead to shifts in a large suite of traits that attract pollinators, such as the positioning of anthers and stigma, UV-light response, and corolla color (Hermann et al. 2015; Sheehan et al. 2016; Esfeld et al. 2018), and many times these morphological traits are not associated with each other (Hermann et al. 2013). We missed the information on the hybrids' main pollinator and about which vector promotes the flow of pollen between these species; here, we demonstrated there is no association between floral shape and genetic profile, although corolla color is a good indicator of the main genetic component of the individuals, as indicated in the Structure analysis. A morphometric study using a more comprehensive sampling for these species and putative hybrids that also included the populations outside the contact zones for $P$. axillaris and $P$. exserta showed that hybrids present transgressive variation in their floral shape compared to the parental species. This does not seem to have an impact on their reproductive success, at least based on germinability of their seeds (MC Teixeira unpubl. res.). Thus, we expect a severe impact of introgression on $P$. exserta, at least in the contact zones, and this can increase the survival risk of this species, as previously discussed (Lorenz-Lemke et al. 2006).

We found a high level of inbreeding among individuals of P. axillaris, P. exserta, and putative hybrids, and the analyses of the genetic composition of these individuals confirmed this finding, since the majority of them was assigned as purebred-derivate (see Newhybrids). Backcrosses with parental species, especially with $P$. exserta, and $\mathrm{F} 2$ individuals were also frequently observed, which suggests the persistence of putative hybrids over time. The Newhybrids analysis frequently erroneously sorts backcrosses as parental purebreds (Vähä \& Primmer 2006), which could mean that there is an even higher rate of introgression in both of the species from these contact zones.

This work provides one more piece to the big puzzle that is the process of Petunia speciation in Serra do Sudeste, which is a region where these species have preferentially diverged through changes in pollination syndromes and freely and naturally hybridize. Our results revealed that corolla color is a good indicator of the genetic component of each species and their hybrids, but the shape of the corolla does not always follow the genetic origin or color. In this system, hybridization increased the variability and rate of introgression in both directions.

\section{Acknowledgements}

The authors thank G. Mäder and D.M. Rodrigues for assistance during field collection. This project was supported by the Conselho Nacional de Desenvolvimento Científico e Tecnológico (CNPq) and the Coordenação de Aperfeiçoamento de Pessoal de Nível Superior (CAPES). C.T. was supported by PNPD-CAPES/PPGBM, UFRGS and S.H. received a PDJ-CNPq scholarship.

\section{References}

Abbott RJ, Barton NH, Good JM. 2016. Genomics of hybridization and its evolutionary consequences. Molecular Ecology 25: 2325-2332.

Anderson E, Thompson EA. 2002. A model-based method for identifying species hybrids using multilocus genetic data. Genetics 160: 12171229.

Bossolini E, Klahre U, Brandenburg A, Reinhardt D, Kuhlemeier C. 2011. High-resolution linkage maps of the model organism Petunia reveal substantial synteny decay with the related genome of tomato. Genome 54: 327-340.

Burgarella C, Lorenzo Z, Jabbour-Zahab R, et al. 2009. Detection of hybrids in nature: application to oaks (Quercus suber and Q. ilex). Journal of Heredity 102: 442-452.

Cinget B, Lafontaine G, Gérardi S, Bousquet J. 2015. Integrating phylogeography and paleoecology to investigate the origin and dynamics of hybrida zones: insights from two widespread North American firs. Molecular Ecology 24: 2856-2870.

Cronk Q, Yang JY. 2016. Hybridization between pollination syndromes as an ecological and evolutionary resource. Molecular Ecology 25: 5827-5829.

Earl EA, Holdt BM. 2012. Structure Harvester: a website and program for visualizing Structure output and implementing the Evanno method. Conservation Genetics Resources 4: 359-361. 


\section{Marcelo C. Teixeira, Caroline Turchetto, Sara Hartke, Carolina K. Schnitzler and Loreta B. Freitas}

Esfeld K, Berardi AE, Moser M, Bossolini E, Freitas L, Kuhlemeier C. 2018. Pseudogenization and resurrection of a speciation gene. Current Biology 28: 3776-3786.

Evanno G, Regnaut S, Goudet J. 2005. Detecting the number of clusters of individuals using the software Structure: A simulation study. Molecular Ecology 14: 2611-2620.

Excoffier L, Lischer HEL. 2010. Arlequin suite ver. 3.5: A new series of programs to perform population genetic analyses under Linux and Windows. Molecular Ecology Resources 10: 564-567.

Excoffier L, Smouse PE, Quattro JM. 1992. Analysis of molecular variance inferred from metric distances among DNA haplotypes: application to human mitochondrial DNA restriction data. Genetics 131: 479-491.

Goudet J. 1995. FSTAT (version 1.2): a computer program to calculate F-statistics. Journal of Heredity 86: 485-486.

Gübitz T, Hoballah ME, Dell'Olivo A, Kuhlemeier C. 2009. Petunia as a model system for the genetics and evolution of pollination syndromes. In: Gerats T, Strommer J. (eds.) Petunia evolutionary, developmental and physiological genetics. New York, Springer. p. 29-49.

Hermann K, Klahre U, Moser M, Sheehan H, Mandel T, Kuhlemeier C. 2013. Tight genetic linkage of prezygotic barrier loci creates a multifunctional speciation island in Petunia. Current Biology 23: 873-877.

Hermann K, Klahre U, Venail J, Brandenburg A, Kuhlemeier C. 2015. The genetics of reproductive organ morphology in two Petunia species with contrasting pollination syndromes. Planta 241: 1241-1254.

Imbert E, Wang H, Conchou L, Vincent H, Talavera M, Schatz B. 2014. Positive effect of the yellow morph on female reproductive success in the flower colour polymorphic Iris lutescens (Iridaceae), a deceptive species. Journal of Evolutionary Biology 27: 1965-1974.

Klahre U, Gurba A, Hermann K, et al. 2011. Pollinator choice in Petunia depends on two major genetic loci for floral scent production. Current Biology 21: 730-739.

Klingenberg CP. 2011. MorphoJ: An integrated software package for geometric morphometrics. Molecular Ecology Resources 11: 353-357.

Lorenz-Lemke AP, Mäder G, Muschner VC, et al. 2006. Diversity and natural hybridization in a highly endemic species of Petunia (Solanaceae): a molecular and ecological analysis. Molecular Ecology 15: 4487-4497.

Meier JI, Marques DA, Mwaiko S, Wagner CE, Excoffier L, Seehausen O. 2016. Ancient hybridization fuels rapid cichlid fish adaptive radiations. Nature Communications 8: 14363. doi: 10.1038/ncomms14363

Milano ER, Kenney AM, Juenger TE. 2016. Adaptive differentiation in floral traits in the presence of high gene flow in scarlet gilia (Ipomopsis aggregata). Molecular Ecology 25: 5862-5875.

Oosterhout C, Hutchinson WF, Wills DPM, Shipley P. 2004. MicroChecker: software for identifying and correcting genotyping errors in microsatellite data. Molecular Ecology 4: 535-538.

Overbeck GE, Müller SC, Fidelis A, et al. 2007. Brazil's neglected biome: The south Brazilian campos. Perspectives in Plant Ecology, Evolution and Systematics 9: 101-116.

Pritchard JK, Stephens M, Donnelly P. 2000. Inference of population structure using multilocus genotype data. Genetics 155: 945-59.
Proctor M, Yeo P, Lack A. 1996. The natural history of pollination. Portland, Timber Press.

Reck-Kortmann M, Silva-Arias GA, Segatto ALA, Mäder G, Bonatto SL, Freitas LB. 2014. Multilocus phylogeny reconstruction: new insights into the evolutionary history of the genus Petunia. Molecular Phylogenetics and Evolution 81: 19-28.

Roy A, Franciscaria N, MacKay J, Bousquet J. 1992. Segregating random amplified polymorphic DNAs (RAPDs) in Betula alleghaniensis. Theoretical and Applied Genetics 85: 173-180.

Segatto ALA, Cazé ALR, Turchetto C, et al. 2014. Nuclear and plastid markers reveal the persistence of genetic identity: A new perspective on the evolutionary history of Petunia exserta. Molecular Phylogenetics and Evolution 70: 504-512.

Sheehan H, Moser M, Klahre U, et al. 2016. MYB-FL controls gain and loss of floral UV absorbance, a key trait affecting pollinator preference and reproductive isolation. Nature Genetics 48: 159-166.

Soltis PS, Soltis DE. 2009. The role of hybridization in plant speciation. Annual Review in Plant Biology 60: 561-588.

Stehmann JR, Lorenz-Lemke AP, Freitas LB, Semir J. 2009. The genus Petunia. In: Gerats T, Strommer J. (eds.) Petunia evolutionary, developmental and physiological genetics. New York, Springer. p. 1-28.

Szlachetko DL, Kolanowska M, Muller F, Vannini J, Rojek J, Górniak M. 2017. First Guatemalan record of natural hybridization between Neotropical species of the Lady's Slipper orchid (Orchidaceae, Cypripedioideae). PeerJ 5: e416. doi: 10.7717/peerj.4162

Turchetto C, Fagundes NJR, Segatto ALA, et al. 2014. Diversification in the South American Pampas: the genetic and morphological variation of the widespread Petunia axillaris complex (Solanaceae). Molecular Ecology 23: 374-389.

Turchetto C, Lima JS, Rodrigues DM, Bonatto SL, Freitas LB. 2015a. Pollen dispersal and breeding structure in a hawkmoth-pollinated Pampa grasslands species Petunia axillaris (Solanaceae). Annals of Botany 115: 939-948.

Turchetto C, Segatto AL, Beduschi J, Bonatto SL, Freitas LB. 2015b. Genetic differentiation and hybrid identification using microsatellite markers in closely related wild species. AoB Plants 7: plv084. doi: 10.1093/aobpla/plv084

Turchetto C, Segatto ALA, Silva-Arias GA, et al. 2019. Contact zones and their consequences: Hybridization between two ecologically isolated wild Petunia species. Botanical Journal of the Linnean Society 190: 421-435.

Vähä J, Primmer CR. 2006. Efficiency of model-based Bayesian methods for detecting hybrid individuals under different hybridization scenarios and with different numbers of loci. Molecular Ecology 15: 63-72.

Venail J, Dell'Olivo A, Kuhlemeier C. 2010. Speciation genes in the genus Petunia. Philosophical Transactions of the Royal Society B: Biological Sciences 365: 461-468.

Weir BS, Cockerham CC. 1984. Estimating F-statistics for the analysis of population structure. Evolution 38: 1358-1370.

Yakimowski SB, Rieseberg LH. 2014. The role of homoploid hybridization in evolution: a century of studies synthesizing genetics and ecology. American Journal of Botany 101: 1247-1258. 